\title{
Online coherence identification using dynamic time warping for controlled islanding
}

\author{
Hasan UI BANNA ${ }^{1,2}$, Zhe YU ${ }^{1}$ (D) Di SHI ${ }^{1}$, Zhiwei WANG ${ }^{1}$, \\ Dawei $\mathbf{S U}^{3}$, Chunlei $\mathbf{X U}^{3}$, Sarika Khushalani SOLANKI ${ }^{2}$, \\ Jignesh M. SOLANKI ${ }^{2}$
}

\begin{abstract}
Controlled islanding is considered to be the last countermeasure to prevent a system-wide blackout in case of cascading failures. It splits the system into self-sustained islands to maintain transient stability at the expense of possible loss of load. Generator coherence identification is critical to controlled islanding scheme as it helps identify the optimal cut-set to maintain the transient stability of the post-islanding systems. This paper presents a novel approach for online generator coherency identification using phasor measurement unit (PMU) data and dynamic time warping (DTW). Results from the coherence identification are used to further cluster non-generator buses using spectral clustering with the objective of minimizing power flow disruptions. The proposed approach is validated and compared to existing methods on the IEEE 39-bus system and WECC 179-bus system, through which its advantages are demonstrated.
\end{abstract}

CrossCheck date: 29 June 2018

\begin{tabular}{l}
\hline Received: 10 October 2017 / Accepted: 29 June 2018/Published \\
online: 25 September 2018 \\
@ The Author(s) 2018 \\
Zhe YU \\
zhe.yu@geirina.net \\
Hasan Ul BANNA \\
majorhasan_209@yahoo.com \\
Di SHI \\
di.shi@geirina.net \\
Zhiwei WANG \\
zhiwei.wang@geirina.net \\
Dawei SU \\
543062082@qq.com \\
Chunlei XU \\
chunleixu@sina.com
\end{tabular}
Keywords Coherence identification, Constrained spectral clustering, Controlled islanding, Dynamic time warping, Phasor measurement unit measurement

\section{Introduction}

The expansion of power grid due to regional interconnections and the increase in diversity of the transmission structure owing to ever increasing market competition have made safety and stability of the system operation crucial. Not only disturbances caused by natural calamities such as hurricanes and earthquakes but also operational mistakes may trigger cascading failures, which may result in systemwide blackouts and pose a significant threat to properties and lives [1].

Controlled islanding is a practical approach to prevent system-wide instabilities and blackouts. It splits a power system into smaller subsystems, referred to as islands. The

Sarika Khushalani SOLANKI

solanki@mail.wvu.edu

Jignesh M. SOLANKI

jignesh.solanki@mail.wvu.edu

GEIRI North America, San Jose, USA

2 Department of CSEE, West Virginia University, Morgantown, USA

3 Grid Dispatch Center, State Grid Jiangsu Electric Power Company, Nanjing, China 
objective is to form stable islands by selecting an optimal set of lines to disconnect while minimizing generation/load imbalance, maintaining voltage stability, ensuring generators coherency, and restraining out-of-step oscillations. The stability of each island depends on the coherency of generators. Therefore, correct and adaptive identification of generators coherency is essential. Moreover, the coherency between groups of generators varies over time, due to changing network topology and operating conditions, necessitating real-time coherency determination [2]. With the increasing deployment of phasor measurement units, online measurement-based coherency identification has become feasible.

There is substantial literature on generator coherency identification. A model based continuation method was discussed in [3], which demonstrated that generator coherence changes with system network topology and operating conditions. Another model-based eigenvalue analysis approach was presented in [4]. However, both approaches required precise knowledge of system models, parameters, and states, which are generally difficult to obtain in practice. The slow coherency-based analysis proposed in [5] was an offline model-based approach. Variations in system's conditions and topology may change the grouping of coherent generators. This behavior is associated with the generators loose coherency characteristic, and one coherent generator group may split into subgroups, or multiple coherent groups may join a bigger coherent group [6]. For this reason, identifying coherent generator groups during real-time operation, based on the current system conditions, is preferred as compared to defining it offline. In addition, the slow coherency-based analysis is based on the linearized electromechanical model of a given system, which may fail under certain conditions due to high non-linearity of power systems.

In [7], coherent generator groups were identified using discrete Fourier analysis. Internal voltage phasors of generators were estimated by using voltage and current phasors measured by phasor measurement units (PMUs) at generator terminals. Jonsson et al. further improved this method by combining generator speed with Fourier analysis [8] where Inter-area dominant modes were identified as Fourier coefficients with the most significant amplitude. However, Fourier analysis based approaches assume linearity and stationarity of the data, which does not hold for inter-area oscillations. Principal component analysis (PCA) method proposed in [9] employed bus voltage angle and generator speed for coherency identification but required prior information of system dynamic characteristics, which is difficult to obtain. A correlation coefficient based method as proposed in [10] did not require prior information as PCA method did, but a threshold to identify the correct number of coherent groups, which may vary for different operating conditions and fault locations. The threshold requires expert system knowledge which makes practical implementation of the approach challenging. Another method in [11] used bioinformatics clustering technique which required pre-specification of the number of clusters. Ariff et al. presented an approach based on independent component analysis [12]. Another measurement-based approach, using the artificial neural network (ANN) was introduced in [13] which needed excessive offline training. For large interconnected networks, consideration of all possible groups for offline training is daunting. In addition, the real-time and large volume of PMU data interchange brings forth additional complications in case of communication failure. Succinctly an algorithm that can achieve online coherency with limited system knowledge and is robust to partial data loss is the need of the hour.

In view of this, the paper proposes an approach for online coherency determination that also handles partial observability of the system. It provides an adaptive option to system operators for intentional islanding operation to minimize the impact of cascading outages. The enormous success of dynamic time warping [14-16] for pattern matching tasks encourages its use for generators clustering. The proposed approach has been compared and contrasted with correlation [17] and community detection [2] based approaches for the IEEE 39-bus system. It has also been compared with hierarchical clustering (HC) [18] and ICA [12] for Western interconnection power system in North America (WECC) 179-bus system. Time domain simulations are used to validate and demonstrate the effectiveness of the proposed methodology in minimizing impacts of cascading outages and system-wide blackouts.

The remainder of this paper is organized as follows. Section 2 presents the proposed controlled islanding algorithm and describes the proposed generator coherence identification and non-generator bus clustering approach. In Section 3, the proposal is validated using IEEE 39-bus system and WECC 179-bus system, compared with existing approaches. Section 4 illustrates the capability of dealing with noisy and incomplete data of the proposal, and Section 5 concludes the paper.

\section{Adaptively controlled islanding framework}

Cascading outages can initiate electromechanical oscillations in power systems. As shown in Fig. 1, two cascaded outages occurred at $t=5 \mathrm{~s}$ and $t=7 \mathrm{~s}$, and one generator lost synchronism. The system eventually became unstable at $t=11.45 \mathrm{~s}$. An efficient islanding scheme should separate generators with different behavior and ensure that coherent generators remain on the same island: (1) to 


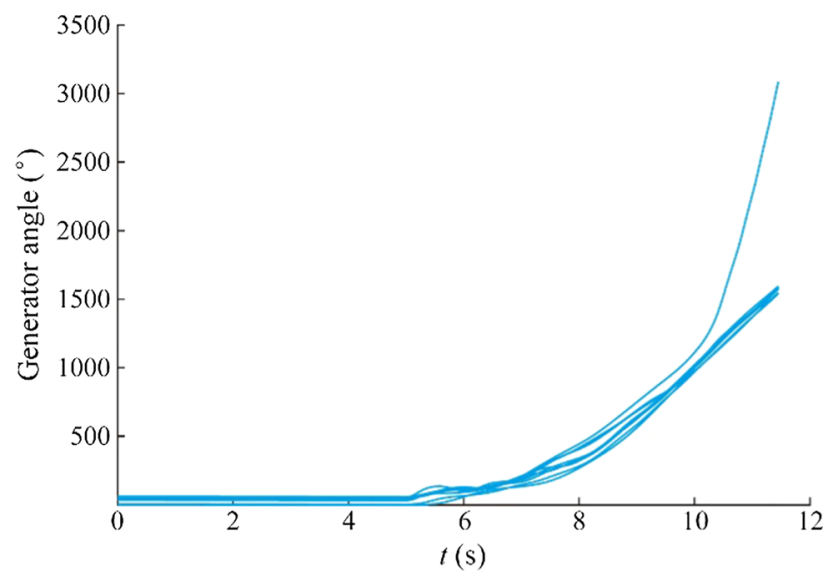

Fig. 1 Generators response following cascaded outages

improve the transient stability; (2) to reduce the chances of further outages.

The proposed adaptive controlled islanding scheme, can be implemented using the following steps.

Step 1: Estimation of generators' rotor angles based on PMU measurements of voltage and current at each generator terminal bus.

Step 2: Similarity evaluation between generators rotor angle responses using algorithm proposed in Section 3.1. It defines a matrix of similarity index for each pair of generators.

Step 3: Optimal number of coherent groups $(k)$ selection by minimizing inter-coherent group distances [19]. It provides the number of unique coherent groups.

Step 4: Grouping of generators using $k$-means into $k$ coherent groups, obtained from Step 3, and building a coherency constraint matrix $\boldsymbol{Q}$ using (8).

Step 5: Building edges' weight matrix $\boldsymbol{W}$ and Laplacian matrix $\boldsymbol{L}$ using (4) and (5) respectively.

Step 6: Formatting the grid as a graph $G=(\boldsymbol{V}, \boldsymbol{E}$, $\boldsymbol{W})$ using power flow results.

Step 7: Solving constrained optimization problem in (7) by finding eigenvalues in (8).

Step 8: Ignore eigenvectors associated with non-positive eigenvalues. After normalizing the remaining eigenvectors, only consider those eigenvectors, which are associated with the smallest $k-1$ eigenvalues.

Step 9: Allocation of non-generator buses to generator groups using the $k$-medoids algorithm on the matrix consists of $k-1$ eigenvectors. The opening of all circuit breakers installed on lines whose terminal buses are in distinct groups will eventually form the desired islands.
Next, each step of the algorithm is explained in detail.

\subsection{Dynamic time warping (DTW) based generator coherency identification}

Generator coherency identification is primarily a similarity matching problem. Therefore, a method from pattern recognition field can be employed. Several similarity measures have been presented in the pattern recognition field including Euclidean distance, Hausdorff, dynamic time warping, Pearson correlation, Mahalanobis, etc. [14, 15]. However, out of these similarity measures, dynamic time warping provides a non-linear mapping between trajectories by minimizing the distance between them [16]. Due to its better performance for partial observability, dynamic time warping was implemented in this paper for generator coherency determination.

When a disturbance occurs in a power system, the generators' responses are governed by their inertia and location in the system. Some generators exhibit similarity in their time domain responses thus are considered as coherent and can be clustered. Here, rotor angle response is selected as the metric for generator coherence identification. For example, generator $p$ and $q$ are considered coherent if $\Delta \delta_{p}(t)-\Delta \delta_{q}(t) \approx 0$ or $\Delta \delta_{p}(t)-\Delta \delta_{q}(t)=$ constant, where $\Delta \delta_{p}(t)$ and $\Delta \delta_{q}(t)$ are the deviations of rotor angles of generator $p$ and $q$, respectively [20]. In this section, the DTW technique is proposed to identify the similarity between rotor angle responses of generators in the system.

Given voltage and current phasor measurements at generator terminal buses, rotor angle responses of these generators $\delta$ can be estimated using least squares (LS) or Kalman filter (KF) based approaches [21]. Consider two rotor angle trajectories $\delta_{p}=\left\{\delta_{p 1}, \delta_{p 2}, \ldots, \delta_{p i}\right\}$ and $\delta_{q}=$ $\left\{\delta_{q 1}, \delta_{q 2}, \ldots, \delta_{q k}\right\}$ estimated over the same period, where $i$ and $k$ are numbers of data points for generators $p$ and $q$, respectively. Normally $i$ and $k$ are equal. When there is data loss or significant communication delays in PMU data transmission, $i$ and $k$ may be different, and DTW can still handle the data.

A local distance measure $d\left(\delta_{p m}, \delta_{q n}\right)$ of data points $m$ and $n$ from rotor angle trajectories $\delta_{p}$ and $\delta_{q}$ respectively is defined as:

$d\left(\delta_{p m}, \delta_{q n}\right)=\left\|\delta_{p m}-\delta_{q n}\right\|^{2}$

where $m \in\{1,2, \ldots, i\}$ and $n \in\{1,2, \ldots, k\}$. Similarly, a distance matrix $\boldsymbol{D}\left(\delta_{p}, \delta_{q}\right)$ of size $i$-by- $k$ is constructed by calculating local distance measures of each pair of data points from trajectories $\delta_{p}$ and $\delta_{q}$. 


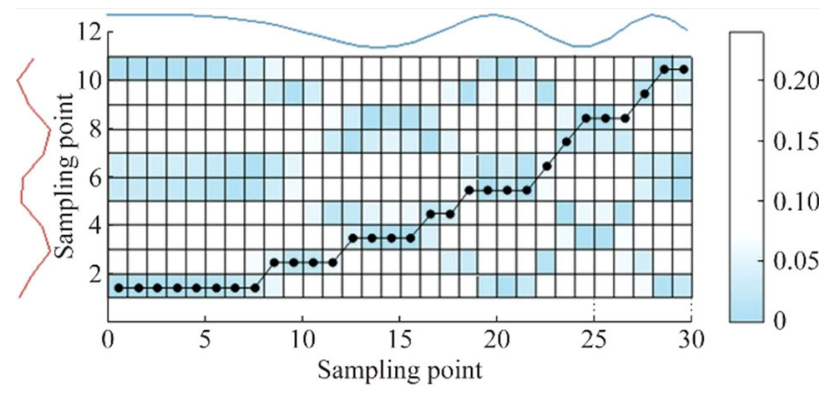

Fig. 2 Optimal warping path

Define $w=\left\{w_{1}, w_{2}, \ldots, w_{L}\right\}$ as a warping path, where $w_{l}$ $=\left(m_{l}, n_{l}\right) \in[1: i] \times[1: k]$ represents the cell in the $m_{l}$ th row, $n_{l}$ th column of a distance matrix $\boldsymbol{D}\left(\delta_{p}, \delta_{q}\right)$. A warping path example of the red and blue trajectories is shown in Fig. 2. A valid warping path satisfies the following conditions as stated in [22].

1) Boundary condition: a valid warping path starts from one corner of the distance matrix $\boldsymbol{D}\left(\delta_{p}, \delta_{q}\right)$ and ends at the diagonally opposite corner, i.e., $w_{1}=(1,1)$ and $w_{L}$ $=(i, k)$.

2) Continuity: a valid warping path is made of adjacent cells (including diagonally adjacent cells), i.e., $w_{l}=(a$, $b)$ and $w_{l-1}=\left(a^{\prime}, b^{\prime}\right), a-a^{\prime} \leq 1$ and $b-b^{\prime} \leq 1$.

3) Monotonicity: a warping path is monotone, i.e., if $w_{l}=$ $(a, b)$ and $w_{l-1}=\left(a^{\prime}, b^{\prime}\right), a-a^{\prime} \geq 0$ and $b-b^{\prime} \geq 0$.

The total distance $d_{w}\left(\delta_{p}, \delta_{q}\right)$ of a warping path $w$ is defined as:

$d_{w}\left(\delta_{p}, \delta_{q}\right)=\sum_{l=1}^{L} d\left(\delta_{p m_{l}}, \delta_{q n_{l}}\right)$

The DTW distance between two trajectories $\delta_{p}$ and $\delta_{q}$ is defined as the minimum total distance among all possible warping paths, which can be found by dynamic programming [22].

$\operatorname{DTW}\left(\delta_{p}, \delta_{q}\right)=d_{w *}\left(\delta_{p}, \delta_{q}\right)=\min \left\{d_{w}\left(\delta_{p}, \delta_{q}\right)\right\}$

In this paper, the similarity between rotor angle responses of generators $p$ and $q$ is represented by $\operatorname{DTW}\left(\delta_{p}, \delta_{q}\right)$. This allows a non-linear mapping between two rotor angle curves, even with data loss or communication delays. DTW is highly ranked in pattern recognition and computer vision fields. It has been widely used in time series analysis, (partial) shape matching, speech recognition, and online signature verification [23]. In $[24,25]$, DTW is tested against Euclidean distance for small data size and is found to provide smaller out-ofsample error rate as a result of its improved similarity metric. A comprehensive explanation of step by step implementation of the DTW algorithm has been presented with an example in Appendix A.

Given the coherency of generators, the optimal number of coherent groups $k$ is selected by minimizing inter-coherent group distances [19]. Further, having the number of coherent groups of generators, various clustering methods, such as $k$-means clustering [26], can be employed to group generators.

\subsection{Buses clustering for controlled islanding}

After clustering generators, the next step is to find an optimal cut set for controlled islanding with generator coherency information as a constraint. The main task is to allocate non-generator buses to coherent generator groups based on a certain metric, which is minimum power flow disruption in this paper.

Several techniques are present in literature for this "where to island" problem including graph clustering. In [27], a $k$-way partitioning algorithm was proposed which partitions the power network into islands by optimizing minimum load generation imbalance. A kernel $k$-means multi-level technique is presented to create islands based on minimum power flow disruption [28]. Both methods are computationally efficient. However, neither of these two methods considers the system's dynamic constraints. Furthermore, neglecting generator dynamic behavior may partition the power network into unstable islands. A particle swarm optimization-based angle modulated algorithm is presented in [29] and utilized minimum load generation imbalance to obtain an islanding solution. Reference [30] presents a Krylov based method to minimize load generation imbalance for islanding. Further, the computational complexity of techniques presented in [29, 30] is reduced by neglecting connectivity of sub-graphs or solving for a simplified network. However, the islanding solutions may contain isolated buses or some solutions, which are lost after simplification, could be better than the solution obtained by the algorithm [31]. The spectral clusteringbased approach is used to solve the "where to island" problem in this paper, which builds on the concept of minimum graph-cut [32]. The objective is to minimize the power flow disruption subject to the generator grouping from Section 2.1. The minimum graph cut formulation is stated as follows.

Power network can be represented as a weighted graph $G=(\boldsymbol{V}, \boldsymbol{E}, \boldsymbol{W})$ with vertices $(\boldsymbol{V})$ and edges $(\boldsymbol{E})$ resembling buses and branches (lines or transformers), respectively. To replicate characteristics of the power grid, each edge in the graph is assigned a certain weight $(\boldsymbol{W})$, which can be any system parameter depending on the targeted application. In this work, power flows through branches are used as the weighting factors. Further, to accommodate system losses, 
weights are evaluated by averaging power flows measured at both sides of the lines as follows.

$W_{i j}=W_{j i}= \begin{cases}\left(\left|P_{i j}\right|+\left|P_{j i}\right|\right) / 2 & i \neq j \\ 0 & i=j\end{cases}$

where $P_{i j}$ and $P_{j i}$ are the active power flows measured at terminal $i$ and $j$ of branch $i-j$, respectively. The weight matrix in (4) considers the dynamic characteristic of the power network as power flow changes with system operating conditions. After evaluating the weight matrix, an un-normalized Laplacian matrix, $\boldsymbol{L}$, can be formulated with its element $L_{i j}$ calculated as:

$L_{i j}= \begin{cases}-W_{i j} & i \neq j \\ d_{i}=\sum_{j=1}^{n} W_{i j} & i=j\end{cases}$

where $d_{i}$ is the sum of weights of all edges connected to node $i$. To make graphs with different weights comparable, the Laplacian matrix can be normalized as $\boldsymbol{L}_{N}=\boldsymbol{D}^{-1 / 2} \boldsymbol{L} \boldsymbol{D}^{-1 / 2}$ [33], where $\boldsymbol{D}$ is a diagonal degree matrix with $d_{i}$ as its diagonal entries.

Given the coherent generator groups, we apply spectral clustering to further cluster buses for controlled islanding. To incorporate generator coherency information as a constraint in spectral clustering, two types of linkages can be introduced: must link (ML) and cannot link (CL). ML constraints ensure the coherent generators remain on the same island while CL keeps the non-coherent generators on different islands. A linkage constraint matrix $\boldsymbol{Q}$ is defined as:

$Q_{i, j}= \begin{cases}+1 & i, j \in M L \\ -1 & i, j \in C L \\ 0 & \text { else }\end{cases}$

Let $\boldsymbol{u} \in\{-1,+1\}^{N}$ be an island indicator vector for $N$ buses, where $u_{i}=+1$ if bus $i$ belongs to island + and $u_{i}=$ -1 if bus $i$ belongs to island - . An index $\boldsymbol{u}^{\mathrm{T}} \boldsymbol{Q u}=$ $\sum_{i j} u_{i} u_{j} Q_{i j}$ can be defined to determine how well constraints in $\boldsymbol{Q}$ are satisfied by the assignment $\boldsymbol{u}$. The greater the value of $\boldsymbol{u}^{\mathrm{T}} \boldsymbol{Q u}$ is, the more satisfied the coherency constraints $\boldsymbol{Q}$ are by the associated indicator vector $\boldsymbol{u}$ [34]. Variables $u_{i}$ and $\boldsymbol{Q}$ can be extended to more than two islands as $\boldsymbol{u} \in \mathbf{R}^{N}$ and $\boldsymbol{Q} \in \mathbf{R}^{N \times N}$ respectively. If $Q_{i j}>0$, then buses $i$ and $j$ should be on the same island and if $Q_{i j}<$ 0 buses $i$ and $j$ should be placed on different islands. Similar to the normalized Laplacian matrix, constraint matrix $\boldsymbol{Q}$ can also be normalized as $\boldsymbol{Q}_{N}=\boldsymbol{D}^{-1 / 2} \boldsymbol{Q} \boldsymbol{D}^{-1 / 2}$. Finally, the association of non-generator buses to already identified generator groups can be obtained by solving the following constrained optimization problem [19]:

$$
\left\{\begin{array}{r}
\arg _{\boldsymbol{v}} \min \boldsymbol{v}^{\mathrm{T}} \boldsymbol{L}_{N} \boldsymbol{v} \\
\text { s.t. } \boldsymbol{v}^{\mathrm{T}} \boldsymbol{Q}_{N} \boldsymbol{v}>\beta \\
\boldsymbol{v}^{\mathrm{T}} \boldsymbol{v}=v o l \\
\boldsymbol{v} \neq \boldsymbol{D}^{1 / 2} \mathbf{1}
\end{array}\right.
$$

where $\boldsymbol{v}^{\mathrm{T}} \boldsymbol{L}_{N} \boldsymbol{v}$ is the cost of the spectral cut; $\beta$ is the satisfaction threshold for constraints; $v o l=\sum_{i}^{N} d_{i}$ is the volume measure of the graph. $v^{\mathrm{T}} v=v o l$ is used to normalize $v$ and $\boldsymbol{v} \neq \boldsymbol{D}^{1 / 2} \mathbf{1}$ is used to avoid trivial solutions with 1 as a constant vector whose entries are $1 \mathrm{~s}$. The relaxed island indicator vector $\boldsymbol{u}$ can be recovered from $\boldsymbol{v}$ as $\boldsymbol{u}=\boldsymbol{D}^{-1 / 2} \boldsymbol{v}$. The optimal solution of (7) can be obtained using the Karush-Kuhn-Tucker theorem [35] by solving the following generalized eigenvalue problem:

$\boldsymbol{L}_{N} \boldsymbol{v}=\lambda\left(\boldsymbol{Q}_{N}-\frac{\beta}{v o l} \boldsymbol{I}\right) \boldsymbol{v}$

After normalizing eigenvectors associated with positive eigenvalues using $v \leftarrow \frac{v}{\|v\|} \sqrt{v o l}$ and $k$ being the coherent generator groups obtained through the proposed algorithm, $k-1$ eigenvectors with lowest eigenvalues are selected. Finally, the $k$-medoids algorithm [36] can be applied, on a matrix $\boldsymbol{V}^{*}$ having $k-1$ eigenvectors as columns. It will allocate non-generator buses to $k$ islands.

\subsection{Discussion}

The main scope of this work is to present an approach, which can find more stable islands. An islanding solution based on constrained spectral clustering is proposed which splits the power network into islands using minimum power flow disruption. Additionally, to ensure the dynamic stability of the newly formed islands, DTW based generator coherency information is treated as a constraint during spectral clustering based splitting process [37]. Further, it helps for reconnecting the islands and reduces the complexity [38].

Finding an islanding solution with minimum load generation imbalance is indeed an NP-hard problem and considered as a special form of 0-1 knapsack problem [39]. This is why in this work we considered minimum power flow disruption instead of minimum load generation imbalance as the criterion for spectral clustering. It is a $\mathrm{P}$-problem as can be converted into a minimum-cut problem and hence solved efficiently [40]. Consideration of generator coherency constraints during spectral clustering increases its complexity. However, this increased computational complexity can be overcome using recursive bisection to find island boundaries [41]. Thus, using minimum power flow disruption has the advantage of reducing 
the time complexity from NP-hard to P that makes the proposed DTW based islanding approach computationally efficient.

\section{Simulation results and performance evaluation}

The proposed methodology is validated through dynamic simulations of IEEE 39-bus and WECC-179 systems. Cascading outages are created using DSAT tools. Time domain simulations show how the proposed methodology can help in minimizing the impact of cascading outages and avoiding blackouts.

Proposed DTW based coherency is determined using $t=$ 5-7.5 $\mathrm{s}$ of data and is utilized further during constrained spectral clustering. The islanding scheme will then be available in case it is needed. The severity and stability indices during cascading failure will determine the exact timing of the islanding. In the experimental validation, islanding was deployed at $t=9 \mathrm{~s}$ to realize the practical situation where closed-loop algorithms may try to bring the system back towards stability and if those algorithms fail then as a last resort islanding can be adopted to avoid further failure. There is no other reason for the islanding time selection of $t=9 \mathrm{~s}$.

Length of the PMU data is critical for the identification results due to cascading failures occurring at different instances as reported in [2]. We do not attempt to duplicate that discussion but rather emphasize the particular advantages DTW has that makes it suitable for applications in generator coherence identification and controlled islanding

The simulation is performed on a 64-bit Intel ${ }^{\circledR}$ Core i7 Central Processing Unit with 3.00 gigahertz speed, 12 gigabytes installed memory (RAM) and 1 terra byte hard disk space. For both case studies, running the proposed algorithm on Matlab takes $1.12 \mathrm{~s}$. It is expected running the same program using $\mathrm{c} / \mathrm{c}++$ will be 50-100 times faster, which satisfies the online application requirements.

Transient stability essentially means that the generators in one island should maintain synchronization after islanding operation. Transient stability of the newly formed islands primarily depends on the coherency of the generators. Silhouette measure can be used to validate the coherent group formation identified by coherency determination algorithms, which is a measure to validate the consistency of the clusters in the data $[42,43]$. Utilizing this index, it can be shown that how strongly generators in one group are coherent and coupled together as compared to those in other coherent groups. It is expressed as:

$$
V_{i}^{S}=\left\{\begin{array}{cc}
\frac{S_{\mathrm{min}, \mathrm{avg}}^{*}(i)-S_{\mathrm{avg}}^{*}(i)}{S_{\mathrm{min}, \mathrm{avg}}^{*}(i)} & S_{\mathrm{avg}}^{*}(i)<S_{\mathrm{min}, \mathrm{avg}}^{*}(i) \\
0 & S_{\mathrm{avg}}^{*}(i)=S_{\mathrm{min}, \mathrm{avg}}^{*}(i) \\
\frac{S_{\mathrm{min}, \mathrm{avg}}^{*}(i)-S_{\mathrm{avg}}^{*}(i)}{S_{\mathrm{avg}}^{*}(i)} & S_{\mathrm{avg}}^{*}(i)>S_{\mathrm{min}, \mathrm{avg}}^{*}(i)
\end{array}\right.
$$

where $S_{\text {min,avg }}^{*}(i)$ is the minimum average dissimilarity of $i$ th generator w.r.t other coherent groups, and $S_{\text {avg }}^{*}(i)$ is the average dissimilarity of $i$ th generator w.r.t all other generators in the same coherent group. A generator with large silhouette value shows that it is strongly coupled with the generators of its coherent group and weakly coupled with the neighboring groups. Typically, silhouette value lies between 1 and -1 . Generators coherency identification is appropriate if most of the generators have large silhouette values. On the other hand, if silhouette values are very small or negative, it shows the possibility of too many coherent groups [44]. The criteria to determine the most suitable coherent group formation is to check the average silhouette value. The coherent groups' formation having higher average silhouette value will indicate more strongly coupled coherent groups [44].

The coherent generator groups' formation with maximum average silhouette value is considered more appropriate coherency identification [45]. Hence, more coherent generator groups formation will surely ensure more transient stability after islanding and will help in healing the system and avoiding a further blackout. A few researchers have recently used this coherency validation criterion for coherent generator groups' formation evaluation. Therefore, it can be regarded as a measure of the stability of the newly formed islands $[44,45]$

We compared stability in the form of silhouette measure, which is independent of the number of clusters/islands. Also, since the system is already recovering from cascading failures, maintaining transient stability is more crucial and focused in this work than maintaining load generation balance. Load generation imbalance is a biproduct and shows an additional benefit that the proposed approach is carrying. Moreover, researchers have shown that loss of load is not directly related to the number of islands formed $[37,44-46]$. The comparisons in $[37,45,46]$ indicate that the loss of load is more for less number of islands formed and the amount of load lost is not directly proportional to the number of islands.

To compare the performance of the proposed approach with existing benchmarks, same "where to island" algorithm, i.e., spectral clustering is used to find the boundaries of the islands; whereas, generator coherency information is determined through DTW, correlation and community detection methods. Islanding solutions are compared 
regarding stability, minimum load generation imbalance and minimum power flow disruption. The coherent group formation with maximum average silhouette value ensures more stability, serving as the primary objective for controlled islanding and also helps to avoid further cascading failures.

\subsection{Case 1: comparison with correlation based method}

In this case, a 3-phase fault is applied on line 17-16 near bus 17 at $t=5 \mathrm{~s}$ and cleared after $150 \mathrm{~ms}$ with the tripping of the corresponding line. Another line 2-1 is tripped at $t=$ $7 \mathrm{~s}$ following a 3-phase fault of $280 \mathrm{~ms}$ duration [17]. These cascading outages eventually lead the system to lose synchronism at $t=12.36 \mathrm{~s}$ as shown in Fig. 3(a). Voltage magnitudes at buses also go very low resulting in a blackout as can be seen in Fig. 3(b).

The loss of synchronism and voltage violations are clear indications that the system should be split. In a practical implementation, the timing of splitting is determined by the

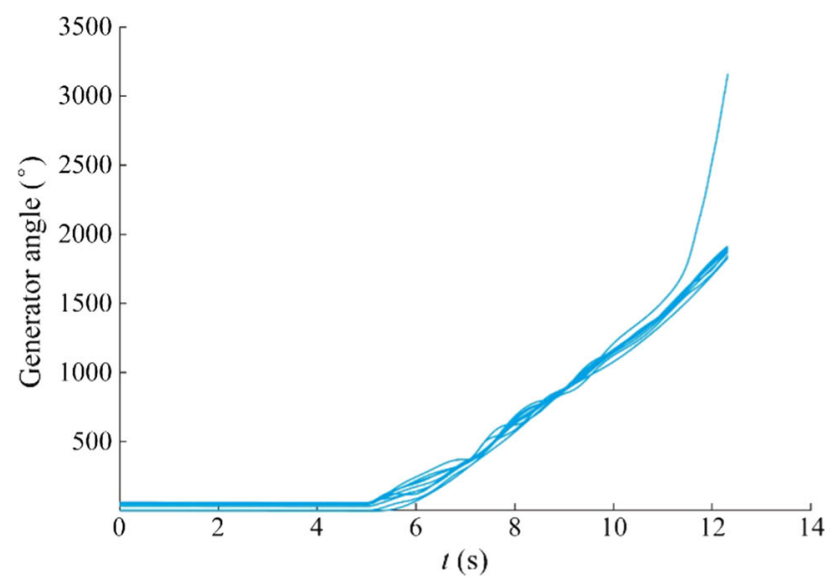

(a)

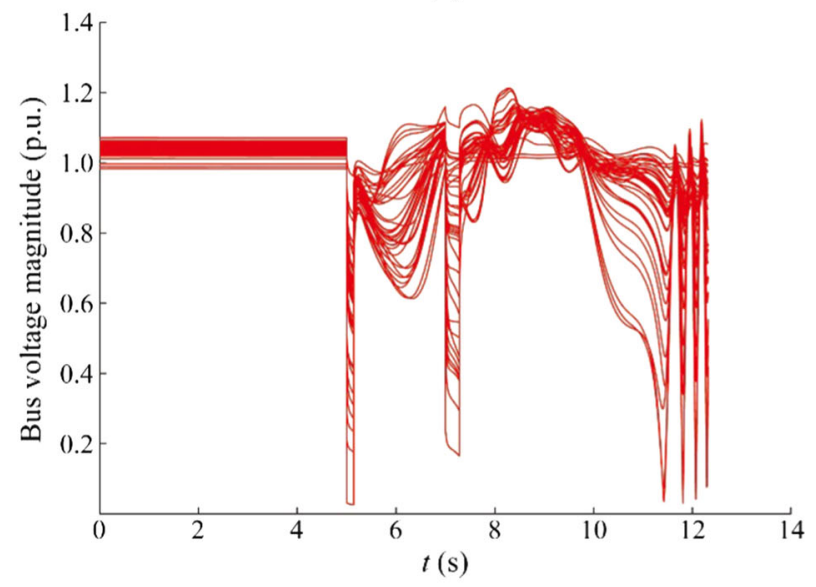

(b)

Fig. 3 System losing synchronism and becoming unstable system operator. Moreover, it depends on the vulnerability analysis performed after severe disturbances [33]. In this paper, we implement intentional islanding at $t=9 \mathrm{~s}$ following two cascading outages. The proposed approach provides a suitable islanding solution using online coherency and pre-fault power flow conditions. The proposed generators coherency algorithm identifies two coherent generator groups as (G1, G8, G9) and (G2, G3, G4, G5, G6, G7). We use this information and solve a constrained spectral clustering problem as described in Section 2.2. Table 1 shows the allocation of non-generator buses to coherent generator groups. It suggests that the breaker on line 3-4 should be opened to form two islands as shown in Fig. 4, and 74.76 MW of active power is disrupted. Generators rotor angles also show the clear formation of two coherent groups after islanding as shown in Fig. 5(a). Voltage magnitude at buses is within limits as can be seen in Fig. 5(b). The numerical results suggest that Algorithm 1 is capable of avoiding system-wide blackouts by keeping voltages at buses within limits and maintaining generators synchronism.

In this case, G10 can be considered as a separate island [37] or as a reference [3, 47] during coherency identification. We adopted the latter approach in this paper. That is why it is not directly considered for DTW based coherency determination. However, its inclusion in the final islanding solution depends on its location and minimum power flow disruption based spectral clustering results.

To check the quality of islanding, active and reactive power generation capacities and load demands are evaluated for each independent island as presented in Table 2 . Generators in each island are capable of fulfilling local demand after islanding. Hence, the proposed online coherency algorithm is capable of identifying suitable generator groups which can be used as dynamic constraints for intentional islanding at the expense of no load shed to avoid a blackout.

The correlation-based method proposed in [17] is carried out as a benchmark. It calculates the correlation coefficient for each pair of generators and splits them based on the average correlation value.

The correlation-based method identifies three coherent groups as (G2, G3), (G4, G5, G6, G7), (G1, G8, G9). The generation capacity of island 1 is below the local demand of the island as shown in Table 3. About 145.1 MW load is shed as shown in Fig. 6(b) with the red color area at the top of the load bar. Further, as shown in Fig. 7, the proposed DTW based approach shows a higher value of the stability measure, hence indicates better tightness for the coherent generators group formation and will be more transiently stable.

Moreover, breakers on lines 3-4 and 14-15 are opened to split the system into three islands. On the other hand, 
Table 1 Allocation of non-generator buses

\begin{tabular}{ll}
\hline Island 1 & Island 2
\end{tabular}

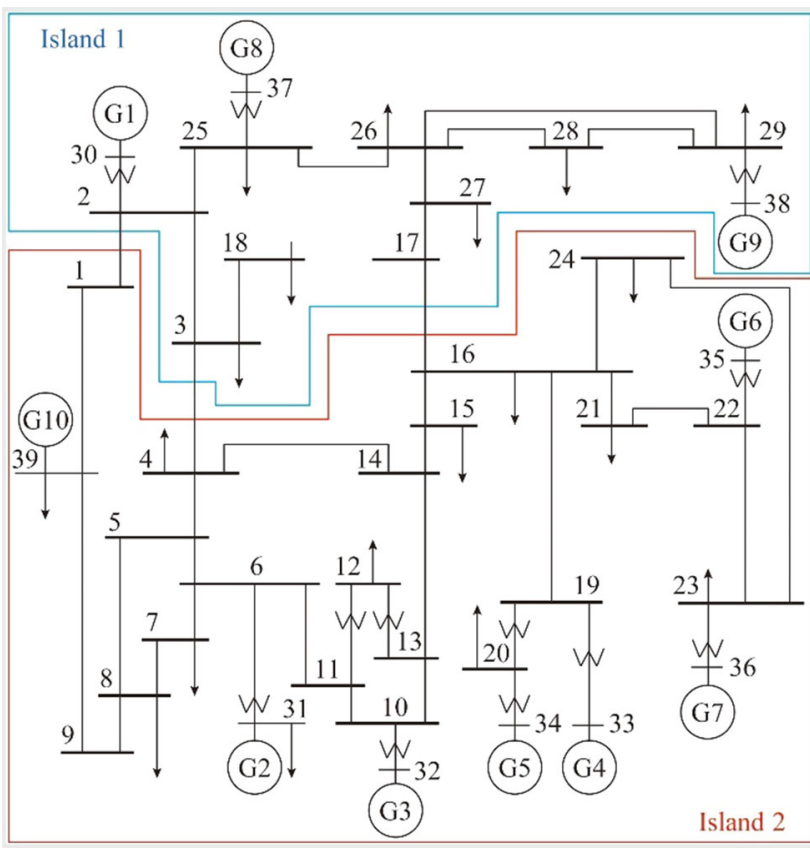

Fig. 4 Formation of two islands

Algorithm 1 sheds no loads with fewer islands and breaker operations. A complete comparison of Algorithm 1 and correlation method based islanding is in Table 4 .

\subsection{Case 2: comparison with community detection method}

A 3-phase fault is simulated on line 13-14 near bus 13 at $t=5 \mathrm{~s}$ and cleared after $150 \mathrm{~ms}$ with the tripping of the line. Another 3-phase fault of 6 cycles duration is simulated in the middle of the line 16-17 at $t=7 \mathrm{~s}$ [2]. Following these cascading outages, the system loses synchronism at $t=11.45 \mathrm{~s}$, and voltage magnitudes also go beyond permissible limits as shown in Fig. 8(a) and Fig. 8(b) respectively if the islanding is not implemented.

The proposed coherency algorithm identifies two generator groups as (G1, G2, G3, G8, G9) and (G4, G5, G6, G7). Solving the constrained spectral clustering problem, we get the allocation of non-generator buses as presented in Table 5. According to the allocation, the breaker on line 14-15 should be opened to split the system into two islands as shown in Fig. 9, and 33.41 MW power is disrupted.

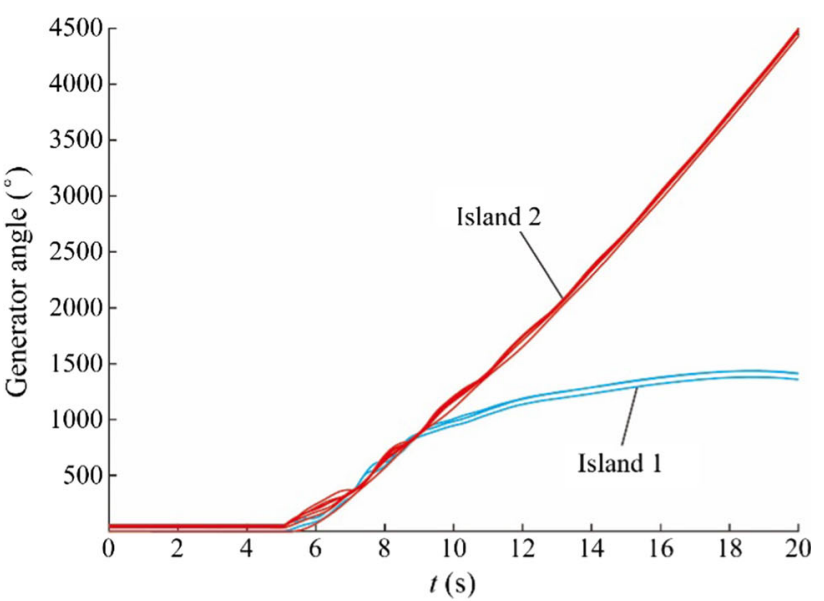

(a)

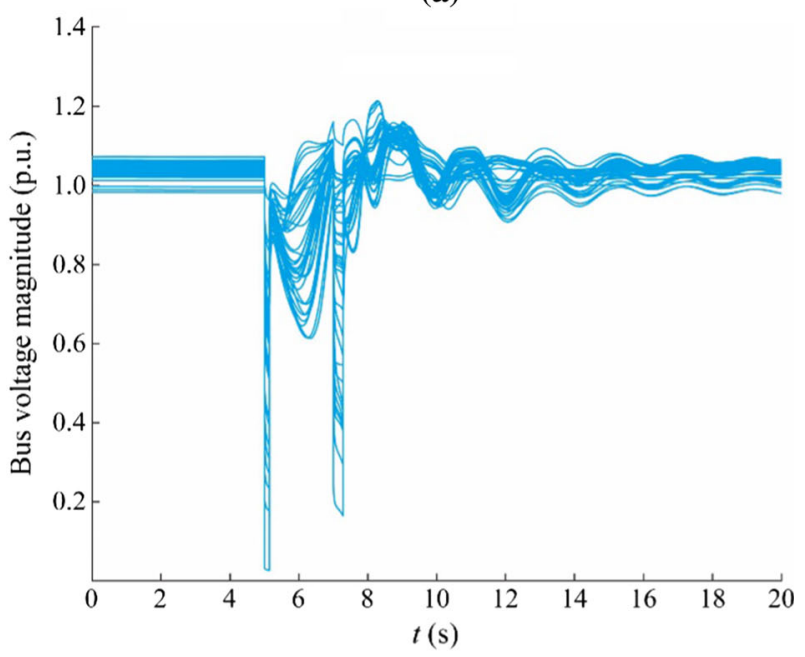

(b)

Fig. 5 Generators rotor angle responses and voltage profiles at system buses after implementing proposed islanding scheme

Rotor angle trajectories shown in Fig. 10(a) indicate the synchronism of generators after islanding. Voltage magnitudes are also within limits as shown in Fig. 10(b).

Active and reactive power generation/load imbalance is evaluated for each island as shown in Table 6. Generators in island 1 are capable of fulfilling the load demand. However, 137.7 MW load is shed in island 2 for stable and balanced operation as shown in Fig. 11(a).

We also carry out the community detection method introduced in [2], and results are summarized in Table 7. Community detection method identifies three coherent generator groups as (G2, G3), (G4, G5, G6, G7), (G1, G8, 
Table 2 Active and reactive power balances in each island using proposed approach

\begin{tabular}{lllll}
\hline Island & $\begin{array}{l}\text { Active power generation capacity } \\
P_{G} \text { (p.u.) }\end{array}$ & $\begin{array}{l}\text { Active power load demand } \\
P_{L} \text { (p.u.) }\end{array}$ & $\begin{array}{l}\text { Reactive power generation capacity } \\
Q_{G} \text { (p.u.) }\end{array}$ & $\begin{array}{l}\text { Reactive power load demand } \\
Q_{L} \text { (p.u.) }\end{array}$ \\
\hline 1 & 16.20 & 16.13 & +24 to -15 & 3.266 \\
2 & 45.73 & 45.36 & +59 to -38 & 14.73 \\
\hline
\end{tabular}

Table 3 Active and reactive power balances in each island using correlation-based algorithm

\begin{tabular}{lllll}
\hline Island & $\begin{array}{l}\text { Active power generation capacity } \\
P_{G} \text { (p.u.) }\end{array}$ & $\begin{array}{l}\text { Active power load demand } \\
P_{L} \text { (p.u.) }\end{array}$ & $\begin{array}{l}\text { Reactive power generation capacity } \\
Q_{G} \text { (p.u.) }\end{array}$ & $\begin{array}{l}\text { Reactive power load demand } \\
Q_{L} \text { (p.u.) }\end{array}$ \\
\hline 1 & 22.239 & 23.69 & +31 to -20 & 7.866 \\
2 & 23.50 & 21.595 & +28 to -18 & 6.858 \\
3 & 16.20 & 16.13 & +24 to -15 & 3.266 \\
\hline
\end{tabular}

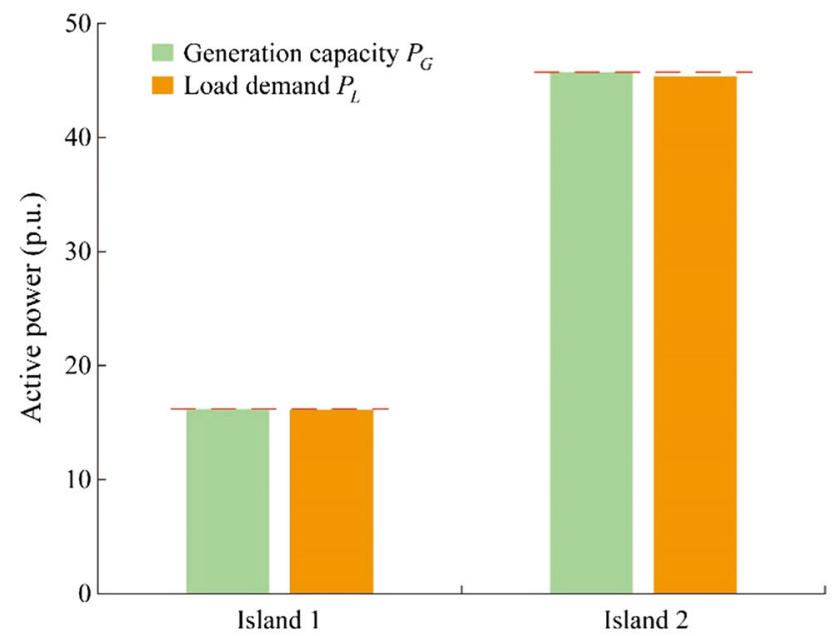

(a) Proposed method

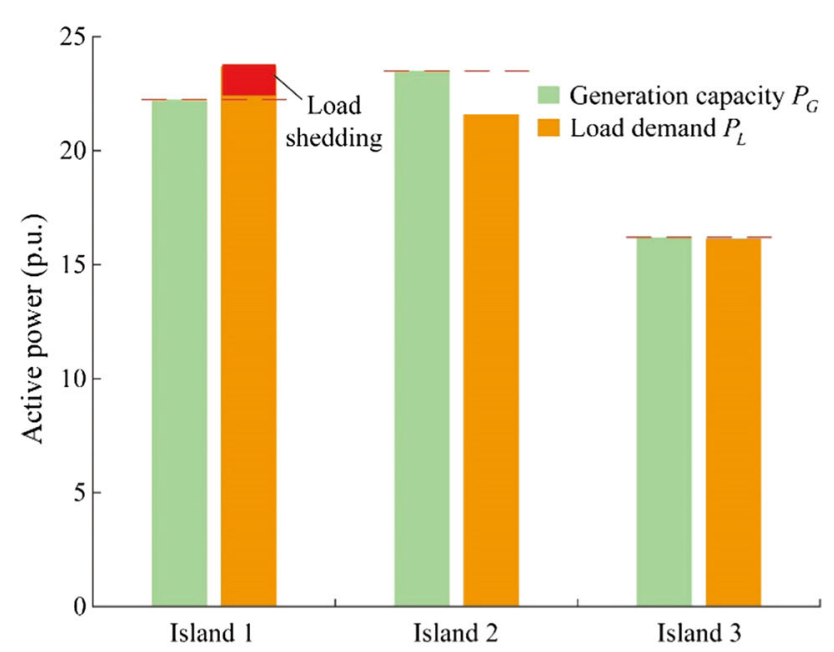

(b) Correlation-based method

Fig. 6 Active power load shedding comparison

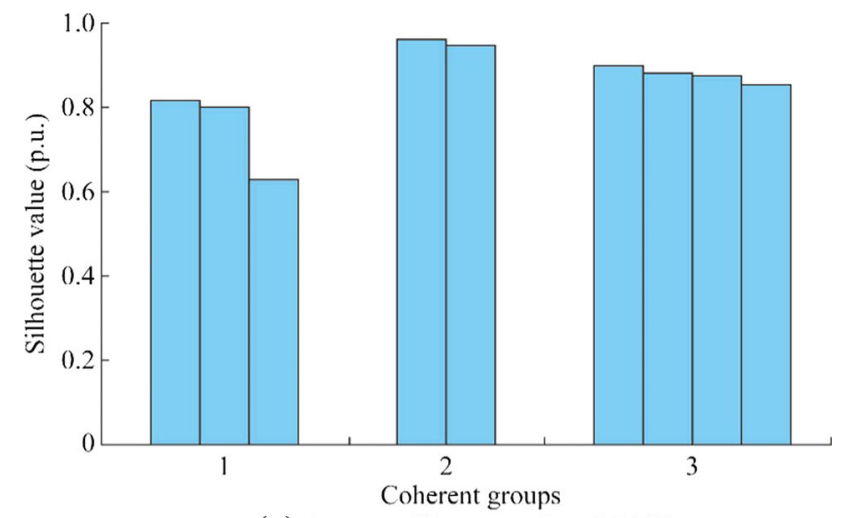

(a) Average silhouette value $=0.8517$

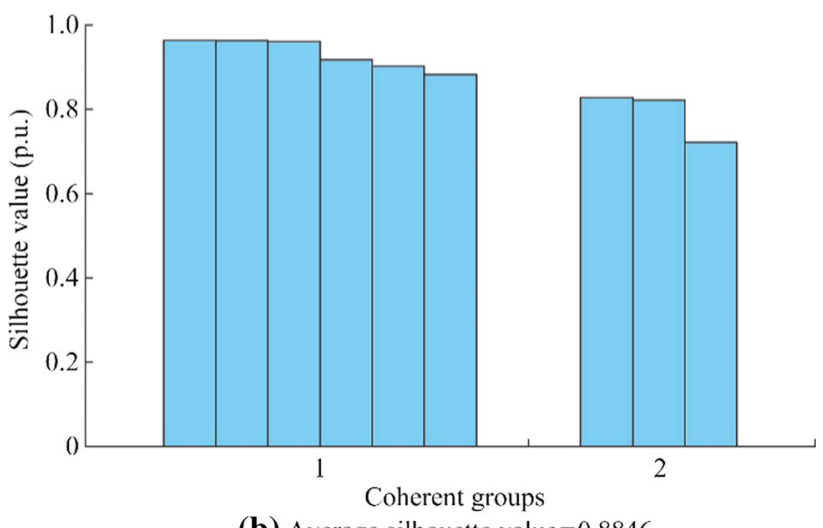

(b) Average silhouette value $=0.8846$

Fig. 7 Silhouette plots for coherent generators groups

G9). The active power generation capacities of island 1 and island 3 are less than the demand of each island. As a consequence, 50.6 MW and 96.43 MW loads are shed in island 1 and 3 respectively as shown in Fig. 11(b). As seen in Fig. 12, the proposed DTW based approach shows a higher value of the stability measure, hence will be more transiently stable. Breakers on lines 3-4, 8-9 and 14-15 are 
Table 4 Performance comparison between proposal and correlation-based algorithms

\begin{tabular}{|c|c|c|c|c|c|}
\hline Method & $\begin{array}{l}\text { No. of switching } \\
\text { operations }\end{array}$ & $\begin{array}{l}\text { Power flow disruption } \\
\text { (MW) }\end{array}$ & $\begin{array}{l}\text { Load/generation imbalance } \\
\text { (MW) }\end{array}$ & $\begin{array}{l}\text { Load shed } \\
\text { (MW) }\end{array}$ & $\begin{array}{l}\text { Silhouette } \\
\text { value }\end{array}$ \\
\hline $\begin{array}{l}\text { Proposed algorithm based } \\
\text { islanding }\end{array}$ & 1 & 74.76 & 44.27 & 0 & 0.8846 \\
\hline $\begin{array}{l}\text { Correlation coefficient based } \\
\text { islanding }\end{array}$ & 2 & 108.17 & 342.6 & 145.1 & 0.8517 \\
\hline
\end{tabular}

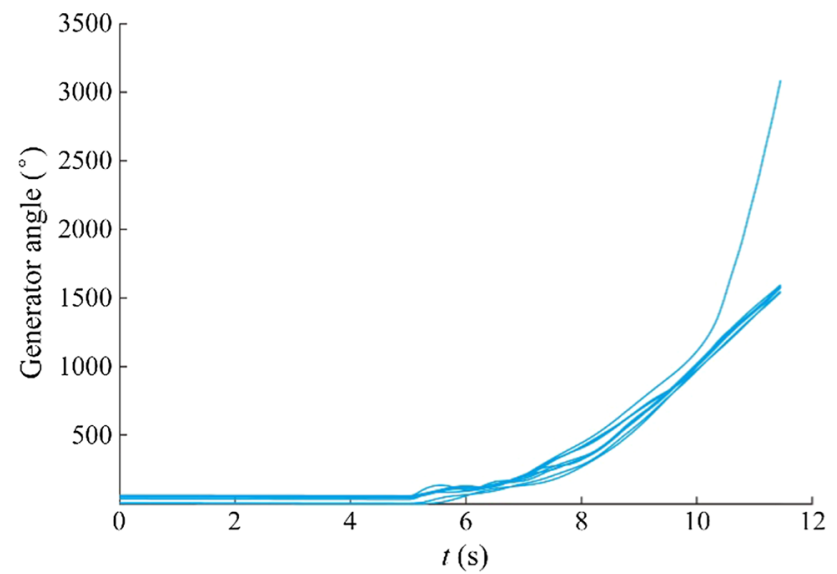

(a)

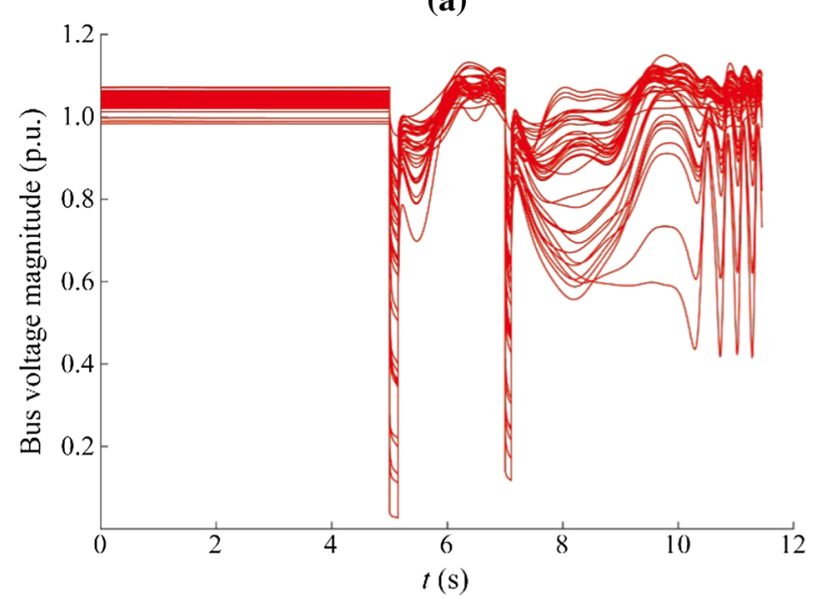

(b)

Fig. 8 System losing synchronism and becoming unstable

opened to split the system into three islands. A complete comparison of Algorithm 1 and community detection method based islanding can be seen in Table 8 , which also indicates superior performance of the proposed algorithm.

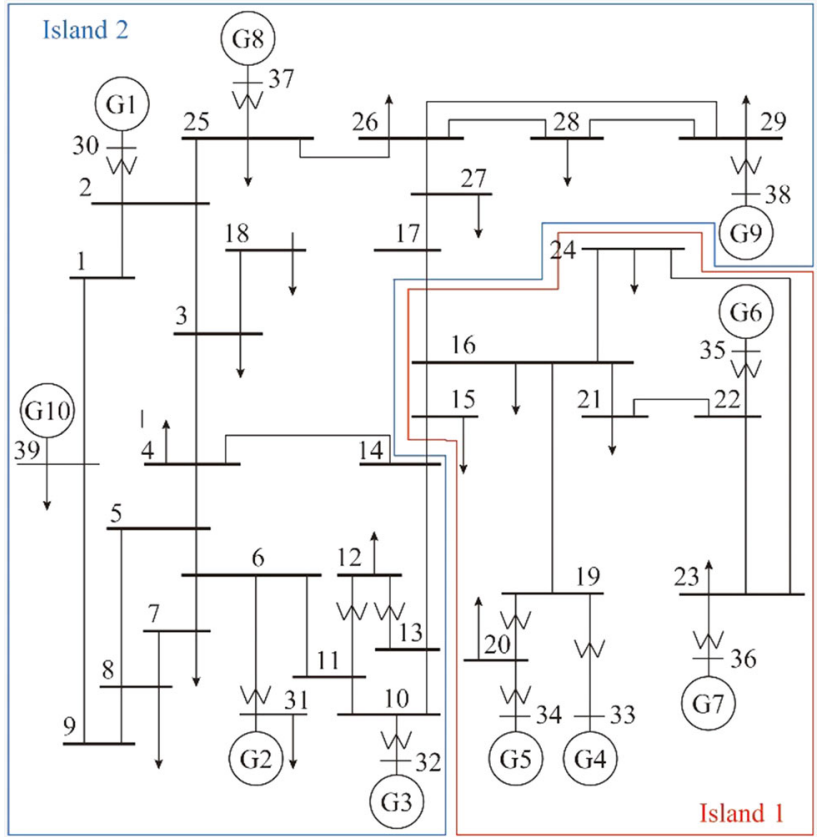

Fig. 9 Formation of two islands

To obtain appropriate islanding, load generation imbalance is not as crucial and critical as ensuring the transient stability within islands as the system is already recovering from cascading outages. Moreover, on an island, load generation imbalance can be compensated through partial load shedding. However, an island with a negative stability margin and good load generation imbalance will collapse. Hence, a proper islanding solution must ensure that coherent generators remain on the same island to improve the stability and reduce the chances of further outages [37].

DTW based coherency determination can still handle the data even if the compared trajectories are of different

Table 5 Allocation of non-generator buses

\begin{tabular}{ll}
\hline Island 1 & Island 2 \\
\hline $15,16,19,20,21,22,23,24,33,34,35,36$ & $1,2,3,4,5,6,7,8,9,10,11,12,13,14,17,18,25,26,27,28,29,30,31,32,37,38,39$ \\
\hline
\end{tabular}




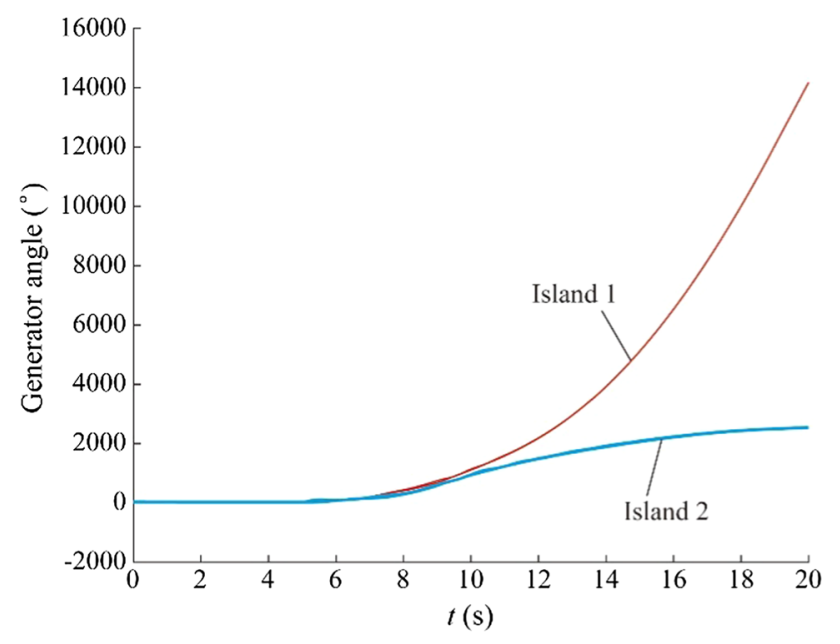

(a)

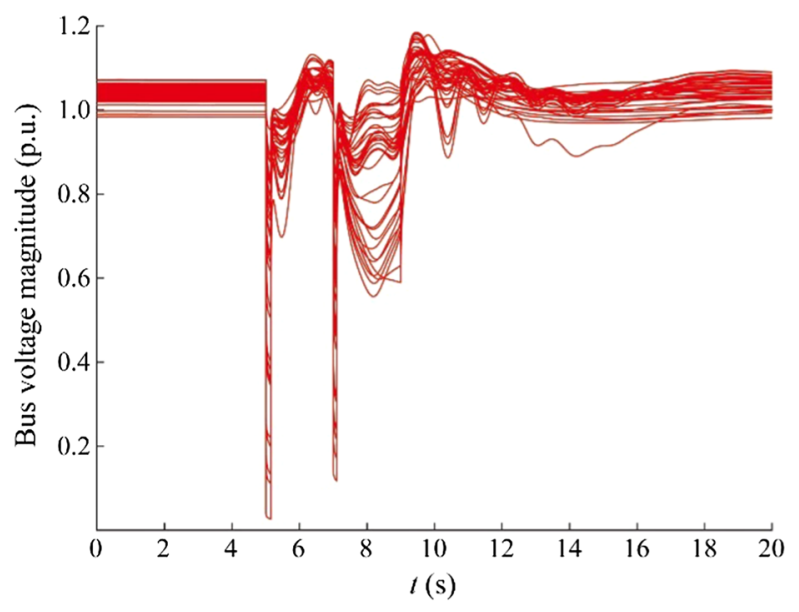

(b)

Fig. 10 Generators rotor angle responses and voltage profiles at system buses after proposed islanding

length; however, since correlation method is a point-topoint distance-based method, it requires an equal length of trajectories to compute the correlation matrix for coherency evaluation. Further, practical application of community detection method is also challenging for online identification of coherent groups with partial observability of the system [2].

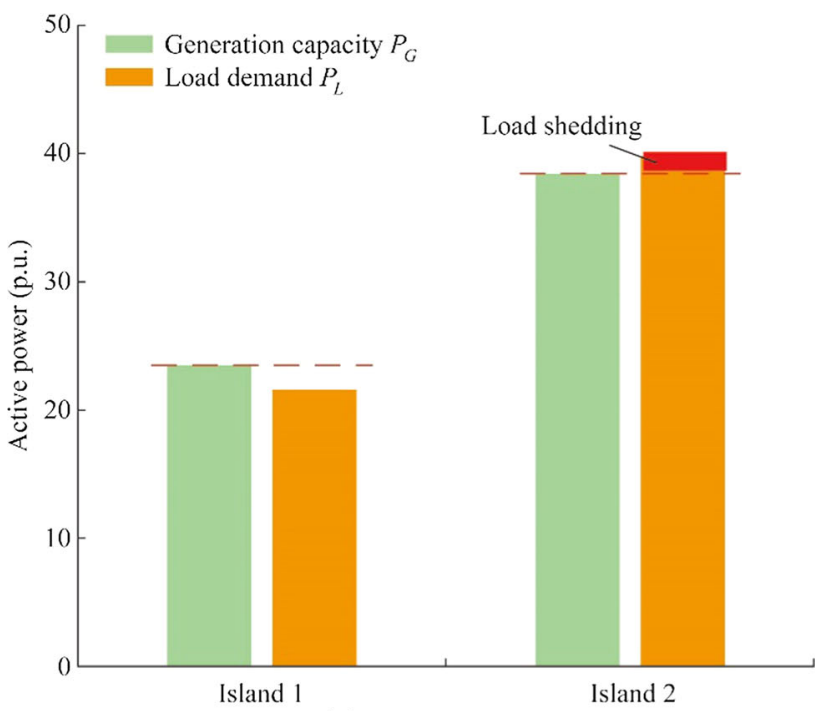

(a) Proposed method

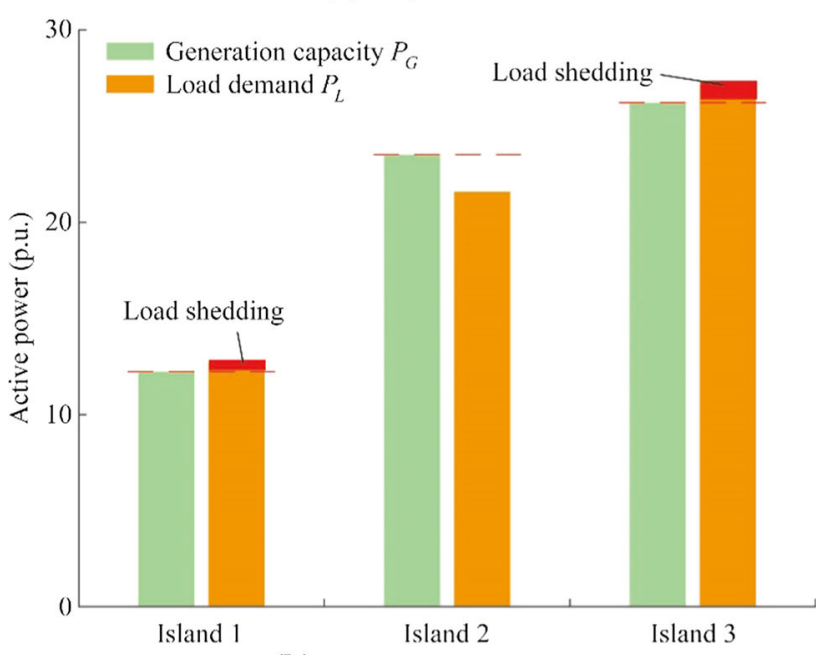

(b) Correlation-based method

Fig. 11 Active power load shedding comparison

\subsection{Western interconnection power system of North America}

A modified version of Western Interconnection power system of North America is also employed to validate and demonstrate the performance of proposed coherency determination method. It has 29 synchronous generators. System's topological structure and parameters can be found in [48]. Rotor angle trajectories' data of nine poorly

Table 6 Active and reactive power balances in each island using proposed approach

\begin{tabular}{lllll}
\hline Island & $\begin{array}{l}\text { Active power generation capacity } \\
P_{G} \text { (p.u.) }\end{array}$ & $\begin{array}{l}\text { Active power load demand } \\
P_{L} \text { (p.u.) }\end{array}$ & $\begin{array}{l}\text { Reactive power generation capacity } \\
Q_{G} \text { (p.u.) }\end{array}$ & $\begin{array}{l}\text { Reactive power load demand } \\
Q_{L} \text { (p.u.) }\end{array}$ \\
\hline 1 & 23.50 & 21.59 & +28 to -18 & 7.16 \\
2 & 38.43 & 39.81 & +55 to -35 & 10.83 \\
\hline
\end{tabular}


Table 7 Active and reactive power balances in each island using community detection based algorithm

\begin{tabular}{lllll}
\hline Island & $\begin{array}{l}\text { Active power generation capacity } \\
P_{G} \text { (p.u.) }\end{array}$ & $\begin{array}{l}\text { Active power load demand } \\
P_{L} \text { (p.u.) }\end{array}$ & $\begin{array}{l}\text { Reactive power generation capacity } \\
Q_{G} \text { (p.u.) }\end{array}$ & $\begin{array}{l}\text { Reactive power load demand } \\
Q_{L} \text { (p.u.) }\end{array}$ \\
\hline 1 & 12.229 & 12.735 & +16 to -10 & 5.366 \\
2 & 23.50 & 21.58 & +28 to -18 & 6.864 \\
3 & 26.21 & 27.175 & +39 to -25 & 5.766 \\
\hline
\end{tabular}

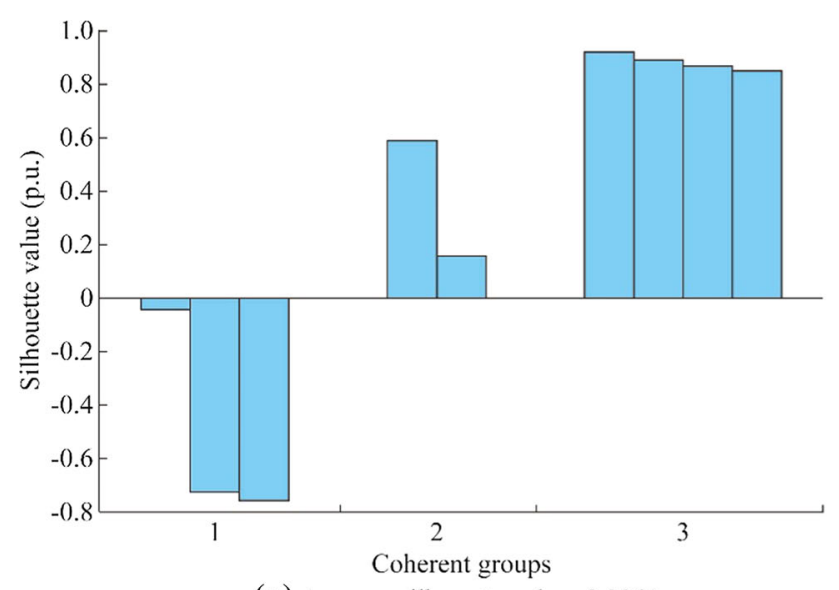

(a) Average silhouette value $=0.3061$

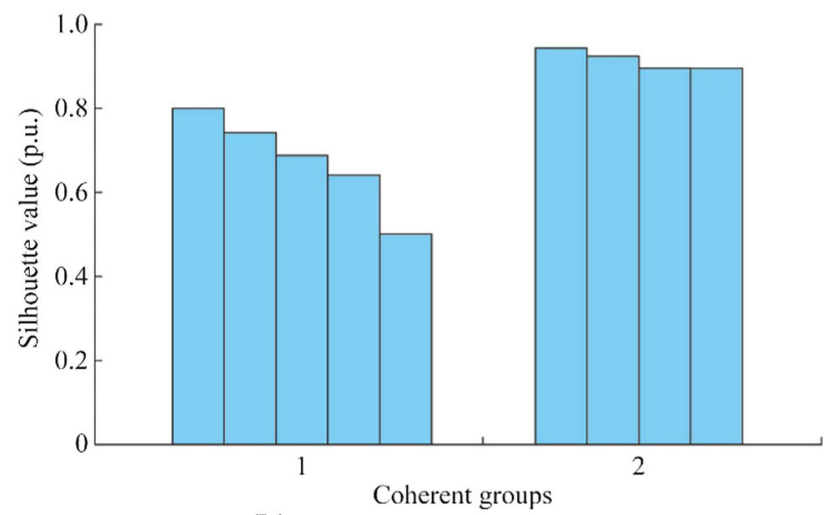

(b) Average silhouette value $=0.7810$

Fig. 12 Silhouette plots for coherent generators groups

damped oscillation cases, named as ND_1, ND_2,..., ND_9, are utilized to signify the performance and comparison of the proposed coherency identification with $\mathrm{HC}$
[18], and ICA [12] methods. Coherency identification results of the proposed, ICA and HC methods along with their average silhouette values are shown in Table 9. Generators' sets are formed as $A=\{103,112,116,118\}$, $B=\{13,15,40,43,47,138,140,144,148,149\}, C=\{30$, $35,65,70,77,79\}, D=\{4,6,9,11,18,36,45,159,162\}$, $D 1=\{4,6,9,11,18\}, D 2=\{36,45,159,162\}[44]$.

Higher value of the metric average silhouette value $\left(V_{i}^{s}\right)$ indicates the better generators' coherency identification. As can be seen from Table 9, the proposed coherency method has a higher average silhouette value of each case than $\mathrm{HC}$ and ICA methods. It signifies that the generators identified by the proposed method are more strongly matched to its coherent group and poorly matched to its neighboring groups. Hence, the proposed coherency method is effective to determine generators' coherency considering the average silhouette value.

The proposed DTW based coherency identification approach can be integrated into the wide area monitoring system in the control center. Coherent generator groups can be determined from PMU measurements and provide widearea monitoring and control for controlled islanding.

\section{Performance of proposed approach with partial observability and noise}

The performance of online PMU measurements-based algorithms is sensitive to partial loss or delay. In PMU based wide area measurement system, communication link failure is common, which may lead the system to be partially observable. Monitoring and control with incomplete information may result in misoperation. Hence, it is

Table 8 Performance comparison between proposed and community detection based algorithms

\begin{tabular}{|c|c|c|c|c|c|}
\hline Method & $\begin{array}{l}\text { No. of switching } \\
\text { operations }\end{array}$ & $\begin{array}{l}\text { Power flow disruption } \\
\text { (MW) }\end{array}$ & $\begin{array}{l}\text { Load/generation imbalance } \\
\text { (MW) }\end{array}$ & $\begin{array}{l}\text { Load shed } \\
\text { (MW) }\end{array}$ & $\begin{array}{l}\text { Silhouette } \\
\text { value }\end{array}$ \\
\hline $\begin{array}{l}\text { Proposed algorithm based } \\
\text { islanding }\end{array}$ & 1 & 33.41 & 328.2 & 137.7 & 0.781 \\
\hline $\begin{array}{l}\text { Community detection based } \\
\text { islanding }\end{array}$ & 3 & 120.94 & 339.03 & 147.03 & 0.3061 \\
\hline
\end{tabular}


Table 9 Comparison of proposed coherency method with HC and ICA method for Western interconnection power system of North America

\begin{tabular}{|c|c|c|c|}
\hline Case & Proposal & HC[48] & ICA[10] \\
\hline ND_1 & $\begin{array}{l}V_{i}^{S}=0.8579 \\
C G 1=D \\
C G 2=\overline{C G 1}\end{array}$ & $\begin{array}{l}V_{i}^{S}=0.5559 \\
C G 1=\{45,159\} \\
C G 2=\overline{C G 1}\end{array}$ & $\begin{array}{l}V_{i}^{S}=0.8502 \\
C G 1=\overline{C G 2+C G 3} \\
C G 2=\{45,159\} \\
C G 3=\{D-C G 2\}\end{array}$ \\
\hline ND_2 & $\begin{array}{l}V_{i}^{S}=0.6519 \\
C G 1=\{36,116,118\} \\
C G 2=\{30,35,65,79\} \\
C G 3=B+D 1 \\
C G 4=\{45,103,159,162\} \\
C G 5=\{70,77,112\}\end{array}$ & $\begin{array}{l}V_{i}^{S}=-0.1717 \\
C G 1=\{65\} \\
C G 2=\overline{C G 1}\end{array}$ & $\begin{array}{l}V_{i}^{S}=0.5782 \\
C G 1=A+D 2 \\
C G 2=B+D 1 \\
C G 3=C\end{array}$ \\
\hline ND_3 & $\begin{array}{l}V_{i}^{S}=0.8024 \\
C G 1=C \\
C G 2=\overline{C G 1}\end{array}$ & $\begin{array}{l}V_{i}^{S}=-0.2438 \\
C G 1=\{6,11\} \\
C G 2=\overline{C G 1}\end{array}$ & $\begin{array}{l}V_{i}^{S}=0.5273 \\
C G 1=\overline{C G 2+C G 3} \\
C G 2=B+D 1 \\
C G 3=\{65\}\end{array}$ \\
\hline ND_4 & $\begin{array}{l}V_{i}^{S}=0.7553 \\
C G 1=\{13,15,138,148\} \\
C G 2=D \\
C G 3=C \\
C G 4=B-G 1\end{array}$ & $\begin{array}{l}V_{i}^{S}=0.3386 \\
C G 1=\{6,11\} \\
C G 2=\overline{C G 1}\end{array}$ & $\begin{array}{l}V_{i}^{S}=0.6221 \\
C G 1=\overline{C G 2+C G 3} \\
C G 2=D-\{6,11\} \\
C G 3=\{6,11\}\end{array}$ \\
\hline ND_5 & $\begin{array}{l}V_{i}^{S}=0.7826 \\
C G 1=C+\{112\} \\
C G 2=\overline{C G 1}\end{array}$ & $\begin{array}{l}V_{i}^{S}=-0.1541 \\
C G 1=\{6,11\} \\
C G 2=\overline{C G 1}\end{array}$ & $\begin{array}{l}V_{i}^{S}=0.5273 \\
C G 1=\overline{C G 2+C G 3} \\
C G 2=B+D 1 \\
C G 3=\{65\}\end{array}$ \\
\hline ND_6 & $\begin{array}{l}V_{i}^{S}=0.8706 \\
C G 1=D \\
C G 2=\overline{C G 1}\end{array}$ & $\begin{array}{l}V_{i}^{S}=0.5735 \\
C G 1=\{45,159\} \\
C G 2=\overline{C G 1}\end{array}$ & $\begin{array}{l}V_{i}^{S}=0.8535 \\
C G 1=\overline{C G 2+C G 3} \\
C G 2=D-\{6,11\} \\
C G 3=\{6,11\}\end{array}$ \\
\hline ND_7 & $\begin{array}{l}V_{i}^{S}=0.8578 \\
C G 1=D \\
C G 2=\overline{C G 1}\end{array}$ & $\begin{array}{l}V_{i}^{S}=0.518 \\
C G 1=\{45,159\} \\
C G 2=\overline{C G 1}\end{array}$ & $\begin{array}{l}V_{i}^{S}=0.8439 \\
C G 1=\overline{C G 2+C G 3} \\
C G 2=D-\{6,11\} \\
C G 3=\{6,11\}\end{array}$ \\
\hline ND_8 & $\begin{array}{l}V_{i}^{S}=0.8533 \\
C G 1=D \\
C G 2=\overline{C G 1}\end{array}$ & $\begin{array}{l}V_{i}^{S}=0.5401 \\
C G 1=\{45,159\} \\
C G 2=\overline{C G 1}\end{array}$ & $\begin{array}{l}V_{i}^{S}=0.8545 \\
C G 1=\overline{C G 2+C G 3} \\
C G 2=D-\{6,11\} \\
C G 3=\{6,11\}\end{array}$ \\
\hline ND_9 & $\begin{array}{l}V_{i}^{S}=0.8698 \\
C G 1=\{70,77,112\} \\
C G 2=B+D 1+\{103\}-\{6,11\} \\
C G 3=\{35,65\} \\
C G 4=\{6,11,45,159,162\} \\
C G 5=\{36,116,118\} \\
C G 6=\{30,79\}\end{array}$ & $\begin{array}{l}V_{i}^{S}=0.1651 \\
C G 1=\{6,11\} \\
C G 2=\overline{C G 1}\end{array}$ & $\begin{array}{l}V_{i}^{S}=0.8575 \\
C G 1=\overline{C G 1} \\
C G 2=B+D 1\end{array}$ \\
\hline
\end{tabular}




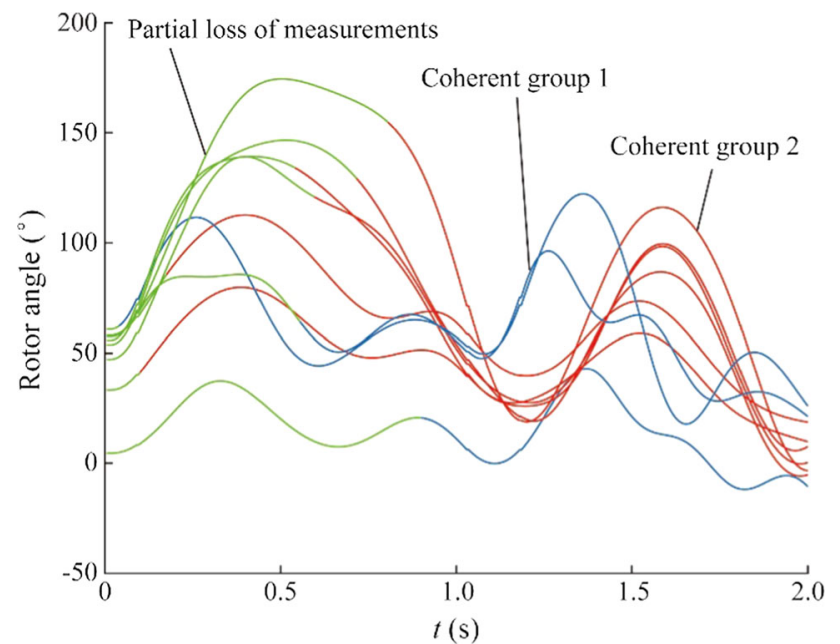

Fig. 13 Performance of proposed coherency algorithm with partial loss of PMU data

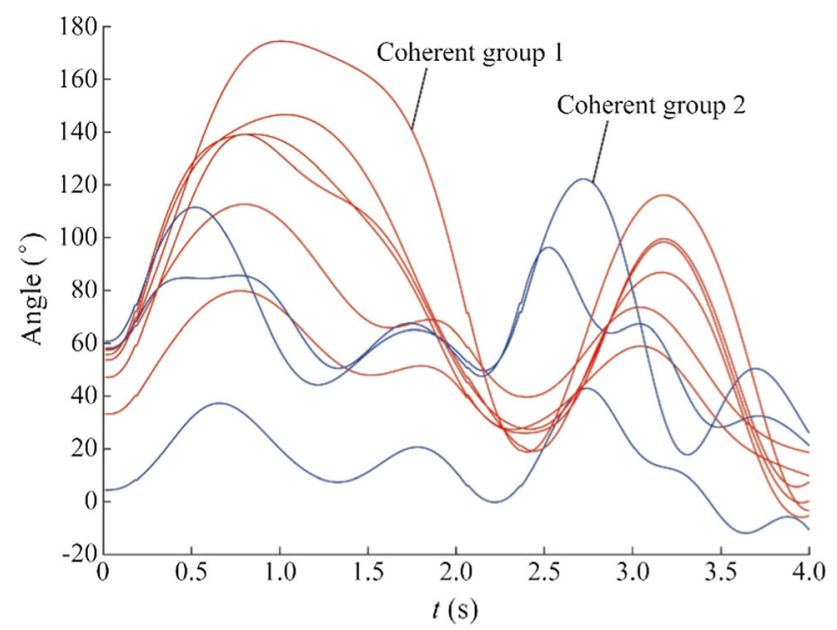

Fig. 14 Generators' coherency grouping identified by proposed coherency method without noise

important to ensure that the coherency identification method is robust to some extent against partial loss/delay of PMU data. Moreover, due to the ever-decreasing cost of PMUs, as compared to benefits gained in the form of increased system observability, their deployment is massively increasing. This increased dependency on PMUs also poses some challenges for online approaches in case of partial observability of the system. This area has not been widely explored, specifically for online coherency identification application. Some researchers also reported it as the limitation of their proposed coherency identification approach [2].

The proposed online coherency approach is applicable in the case of partial observability of the system due to its non-linear nature of similarity computation as explained in

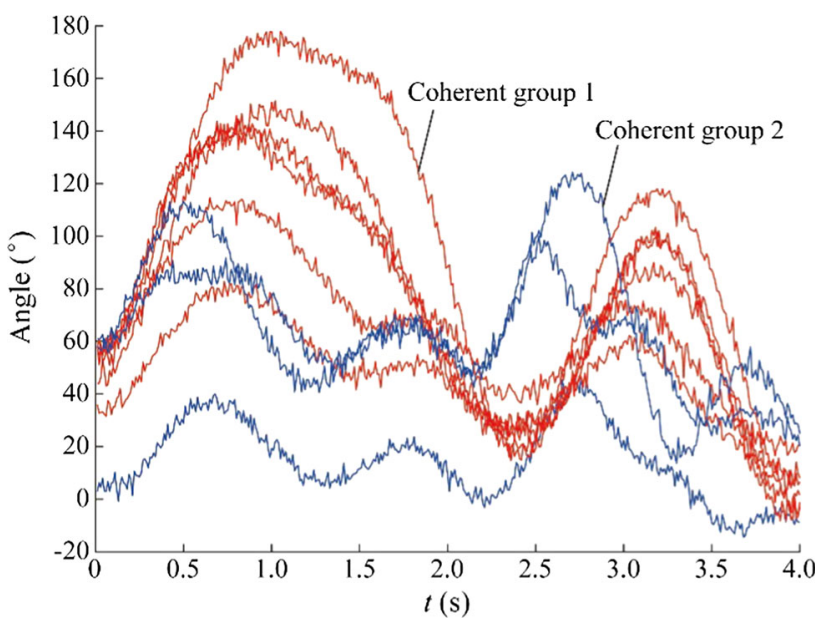

Fig. 15 Generators' coherency grouping identified by proposed coherency method in the presence of white Gaussian noise

Section 2. Consider case 1 mentioned in Section 3, where we have cascaded outages of line 17-16 and 2-1 at $t=5 \mathrm{~s}$ and $t=7 \mathrm{~s}$ respectively. We considered PMUs on generator buses only. To analyze the performance of the proposed online coherency algorithm for a partially observable system, we intentionally remove the initial measurement points for each PMU. Figure 13 shows the experimental results. The green color in each curve indicates the lost part of PMU data. We determine the coherency through the proposed approach. After determining the coherency with such incomplete PMU data, we compare the coherency results with the results obtained without loss of measurements. The algorithm still gives us the same coherency results. Thus, the proposed online coherency approach is robust to a considerable extent for loss/delay of PMU data.

In this paper, we considered the worst-case scenario when a certain consecutive portion of PMU measurements is lost. Measurement loss randomly is an easier problem to solve as compared to the one discussed in this paper. Further, we considered measurement loss rate ranging 5\%45\% for different PMU channels as shown in Fig. 13.

To validate the applicability of the proposed coherency identification method for actual PMU measurements in the presence of noise, white Gaussian noise is added for simulating the measurement noise.

Rotor angle trajectories and their coherency grouping as suggested by the proposed coherency method are shown in Fig. 14. Reference [49] experimentally recommended an SNR value of $45 \mathrm{~dB}$ as a good approximation for real PMU data. However, we test the performance with a higher level of noise, i.e., $30 \mathrm{~dB}$. Figure 15 shows the generator grouping identified by the proposed coherency method in the presence of noise. It can be seen that the coherency 
grouping with white Gaussian noise is the same as the one without noise. Hence, the performance of the proposed method is not affected by a certain level of noise.

\section{Conclusion}

Splitting the system into self-sustained islands is the last resort to maintain transient stability. This paper presents a novel methodology for generator coherency identification. It uses post-fault rotor angle trajectories of generators for coherency determination. For non-generator buses allocation, constrained spectral clustering is applied to minimize power flow disruption, considering coherency matrix as a constraint. Future work includes: (1) allocation of nongenerator buses based on multiple constraints like restoration constraint, thermal limits of transmission lines, etc., in addition to generator coherence constraint; (2) prevention of blackouts using energy storage system without going into islanding operation mode; (3) further testing of the proposed methodology on the real-time simulator for hardware-in-the-loop simulation.

Acknowledgments This work was supported by SGCC Science and Technology Program (No. 5455HJ160007).

Open Access This article is distributed under the terms of the Creative Commons Attribution 4.0 International License (http:// creativecommons.org/licenses/by/4.0/), which permits unrestricted use, distribution, and reproduction in any medium, provided you give appropriate credit to the original author(s) and the source, provide a link to the Creative Commons license, and indicate if changes were made.

\section{Appendix A}

A simple example to further clarify the formulation and implementation of the DTW algorithm is presented here. Consider two trajectories, $\operatorname{tr}_{1}(t)=\{-0.60,-0.65,-0.71$,

\begin{tabular}{|l|c|c|c|c|c|c|c|}
\hline 10 & 0.0961 & 0.1296 & 0.1764 & 0.0841 & 0.0144 & 1.1236 & $\mathbf{4 . 9 7 2 9}$ \\
\hline 9 & 0.3600 & 0.4225 & 0.5041 & 0.3364 & 0.0289 & $\mathbf{0 . 5 9 2 9}$ & 3.7636 \\
\hline 8 & 1.4400 & 1.5625 & 1.7161 & 1.3924 & 0.5929 & $\mathbf{0 . 0 2 8 9}$ & 1.7956 \\
\hline 7 & 3.8416 & 4.0401 & 4.2849 & 3.7636 & 2.3409 & $\mathbf{0 . 3 4 8 1}$ & 0.3364 \\
\hline 6 & 6.5025 & 6.7600 & 7.0756 & 6.4009 & 4.4944 & $\mathbf{1 . 3 9 2 4}$ & 0.0001 \\
\hline 5 & 0.1369 & 0.1764 & 0.2304 & 0.1225 & $\mathbf{0 . 0 0 3 6}$ & 1.0000 & 4.7089 \\
\hline 4 & 0.0484 & 0.0289 & 0.0121 & $\mathbf{0 . 0 5 7 6}$ & 0.4225 & 2.5281 & 7.6176 \\
\hline 3 & 0.0625 & 0.0400 & $\mathbf{0 . 0 1 9 6}$ & 0.0729 & 0.4624 & 2.6244 & 7.7841 \\
\hline 2 & 0.0576 & $\mathbf{0 . 0 3 6 1}$ & 0.0169 & 0.0676 & 0.4489 & 2.5921 & 7.7284 \\
\hline 1 & $\mathbf{0 . 0 7 2 9}$ & 0.0484 & 0.0256 & 0.0841 & 0.4900 & 2.6896 & 7.8961 \\
\hline & 1 & 2 & 3 & 4 & 5 & 6 & 7 \\
\hline
\end{tabular}

Fig. A1 An optimal path formation through a matrix of local distance measures
$-0.58,-0.17,-0.77,1.94\}$ and $t r_{2}(t)=\{-0.87,-0.84,-0.85$, $-0.82,-0.23,1.95,1.360 .60,-0.29\}$. First local distance measures, between each pair of trajectories as formulated in (1), are calculated. A matrix of size $10 \times 7$ is obtained as shown in Fig. A1. Then starting from the element $(1,1)$, multiple summing paths can be traced to reach the final cell $(10,7)$ as described in (2). Out of these possible summing paths, an optimal path which would sum up to minimum distance will represent the DTW distance between trajectories $t r_{1}(t)$ and $t r_{2}(t)$ as expressed in (3) and shown in Fig. A1.

\section{References}

[1] U.S-Canada Power System Outage Task Force (2014) Final report on the August 14, 2003, blackout in the United States and Canada: causes and recommendations. http://www.nerc.com. Accessed 24 Aug 2017

[2] Gomez O, Rios MA (2005) Real time identification of coherent groups for controlled islanding based on graph theory. IET Gen Transm Distrib 9(8):748-758

[3] Wang X, Vittal V, Heydt GT (2005) Tracing generator coherency indices using the continuation method a novel approach. IEEE Trans Power Syst 20(3):1510-1518

[4] Yusof SB, Rogers GJ, Alden RTH (1993) Slow coherency based network partitioning including load buses. IEEE Trans Power Syst 8(3): 1375-1382

[5] You H, Vittal V, Wang X (2004) Slow coherency based islanding. IEEE Trans Power Syst 19(1):483-491

[6] Chow JH (1995) New algorithms for slow coherency aggregation of large power systems. Inst Math Appl 64:95

[7] Ota Y, Ukai H, Nakamura K et al (2002) PMU based midterm stability evaluation of wide-area power system. In: Proceedings of IEEE/PES transmission and distribution conference and exhibition, Yokohama, Japan, 6-10 October 2002, 5 pp

[8] Jonsson M, Begovic M, Daalder J (2004) A new method suitable for real-time generator coherency determination. IEEE Trans Power Syst 19(3):1473-1482

[9] Anaparthi KK, Chaudhuri B, Thornhill NF et al (2005) Coherency identification in power systems through principal component analysis. IEEE Trans Power Syst 20(3):1658-1660

[10] Vahidnia A, Ledwich G, Palmer E et al (2012) Generator coherency and area detection in large power systems. IET Gen Transm Distrib 6(9):874-883

[11] Hashiesh F, Mostafa HE, Helal I et al (2011) Determination of generators coherent groups based on synchrophasors using bioinformatics toolbox. In: Proceedings of IEEE PES conference on innovative smart grid technologies, Jeddah, Saudi Arabia, 17-20 December 2011, 7 pp

[12] Ariff MAM, Pal BC (2013) Coherency identification in interconnected power system - an independent component analysis approach. IEEE Trans Power Syst 28(2):1747-1755

[13] Abd-EI-Rehim MA, Helal ID, Omar MAH (2006) Multi-machine power system dynamic equivalents using artificial intelligence $(\mathrm{ANN})$. In: Proceedings of 11th international middle east power system conference, El-Minia, Egypt, 19-21 December 2006, $11 \mathrm{pp}$

[14] Wang H, Su H, Zheng K et al (2013) An effective study on trajectory similarity measures. In: Proceedings of 24th 
Australian database conference, Darlinghurst, Australia, 13-22 January 2013, $10 \mathrm{pp}$

[15] Zhang Z, Huang K, Tan T (2006) Comparison of similarity measures for trajectory clustering in outdoor surveillance scenes. In: Proceedings of 18th international conference on pattern recognition, Hong Kong, China, 20-24 August 2006, 4 $\mathrm{pp}$

[16] Iglesias F, Kastner W (2013) Analysis of similarity measures in time series clustering for the discovery of building energy patterns. Energies 6(2):579-597

[17] Aghamohammadi MR, Tabandeh SM (2016) A new approach for online coherency identification in power systems based on correlation characteristics of generators rotor angle oscillations. Electr Power Energy Syst 83:470-484

[18] Lin ZZ, Wen FS, Zhao JH et al (2016) Controlled islanding schemes for interconnected power systems based on coherent generator group identification and wide-area measurements. J Mod Power Syst Clean Energy 4(3):440-453

[19] Gonzalez TF (1985) Clustering to minimize the maximum intercluster distance. Theor Comput Sci 38:293-306

[20] Joo SK, Liu CC, Jones LE et al (2004) A coherency and aggregation techniques incorporating rotor and voltage dynamics. IEEE Trans Power Syst 19(2):1068-1075

[21] Fan L, Wehbe Y (2013) Extended Kalman filtering based real time dynamic state and parameter estimation using PMU data. Electr Power Syst Res 103:168-177

[22] Keogh E, Ratanamahatana CA (2005) Exact indexing of dynamic time warping. Knowl Inf Syst 7(3):358-386

[23] Efrat A, Fan Q, Venkatasubramanian S (2007) Curve matching, time warping, and light fields: new algorithms for computing similarity between curves. J Math Imaging Vis 27(3):203-216

[24] Ding H, Trajcevski G, Scheuermann P et al (2008) Querying and mining of time series data: experimental comparison of representations and distance measures. J Proc Very Large Database Endow 1(2):1542-1552

[25] Wang X, Mueen A, Ding H et al (2013) Experimental comparison of representation methods and distance measures for time series data. Data Min Knowl Discov 26(2):275-309

[26] MATLAB (R2017a) Documentation: k-means clustering. http:// www.mathworks.com/help/stats/kmeans.html. Accessed 15 May 2017

[27] Li H, Rosenwald GW, Jung J et al (2005) Strategic power infrastructure defense. Proc IEEE 93(5):918-933

[28] Peiravi A, Ildarabadi R (2009) A fast algorithm for intentional islanding of power systems using the multilevel kernel k-means approach. J Appl Sci 9:2247-2255

[29] Liu L, Liu W, Cartes DA et al (2009) Slow coherency and angle modulated particle swarm optimization based islanding of largescale power systems. Adv Eng Inform 23(1):45-56

[30] Najafi S (2009) Evaluation of interconnected power systems controlled islanding. In: Proceedings of IEEE Bucharest powertech, Bucharest, Romania, 28 June-2 July 2009, 8 pp

[31] Qiao Y (2007) Study on the active splitting control scheme of large-scale power systems. Dissertation, Tsinghua University

[32] Esmaeilian A, Kezunovic M (2017) Prevention of power grid blackouts using intentional islanding scheme. IEEE Trans Ind Appl 53(1):622-629

[33] Tortos JQ, Garcia RS, Brodzki J et al (2015) Constrained spectral clustering based methodology for intentional controlled islanding of large scale power systems. IET Gen Transm Distrib 9(1):31-42

[34] Wang X, Qian B, Davidson I (2014) On constrained spectral clustering and its applications. Data Min Knowl Discov 28(1): $1-30$

[35] Kuhn HW (1982) Nonlinear programming: a historical view. ACM SIGMAP Bull 31:6-18
[36] MATLAB (R2017a) Documentation: k-medoids clustering. http://www.mathworks.com/help/stats/kmedoids.html

[37] Ding L, Gonzalez FM, Wall P et al (2013) Two-step spectral clustering islanding algorithm. IEEE Trans Power Syst 28(1):75-84

[38] Henner VE (1980) A network separation scheme for emergency control. Int J Electr Power Energy Syst 2(2):109-114

[39] Zhao Q, Sun K, Zheng D et al (2003) A study of system splitting strategies for island operation of power system: a two-phase method based on OBDDs. IEEE Trans Power Syst 18(4):1556-1565

[40] Sen A, Ghosh P, Vittal V et al (2009) A new min-cut problem with application to electric power network partitioning. Eur Trans Electr Power 19(6):778-797

[41] Davidson I, Ravi SS (2007) The complexity of non-hierarchical clustering with instance and cluster level constraints. Data Min Knowl Discov 14(1):25-61

[42] Amorim RC, Hennig C (2015) Recovering the number of clusters in data sets with noise features using feature rescaling factors. Inf Sci 324(1):126-145

[43] Rousseeuw PJ (1987) Silhouettes: a graphical aid to the interpretation and validation of cluster analysis. J Comput Appl Math 20:53-65

[44] Lin Z, Wen F, Ding Y et al (2017) Data-driven coherency identification for generators based on spectral clustering. IEEE Trans Ind Inform 14(3):1275-1285

[45] Mahdi M, Istemihan VM (2017) A real time self-healing methodology using model and measurement based islanding algorithms. IEEE Trans Smart Grid. https://doi.org/10.1109/ TSG.2017.2760698

[46] Song H, Wu J, Wu K (2014) A wide-area measurement systemsbased adaptive strategy for controlled islanding in bulk power systems. Energies 7(4):2631-2657

[47] Wang X (2005) Slow coherency grouping based islanding using minimal cutsets and generator coherency index tracing using the continuation method. Dissertation, Iowa State University

[48] Maslennikov S, Wang B, Zhang Q et al (2016) A test cases library for methods locating the sources of sustained oscillations. In: Proceedings of IEEE PES general meeting, Boston, USA, 17-21 July 2016, 5 pp

[49] Brown M, Biswal M, Brahma S et al (2016) Characterizing and quantifying noise in PMU data. In: Proceedings of IEEE PES general meeting, Boston, USA, 17-21 July 2016, 5 pp

Hasan UI BANNA received B.Sc. Engineering from UET Lahore, Pakistan in 2011 and M.S. degree in Power Engineering from Brandenburg Technical University, Germany, in 2014. He did his masters' thesis from SEER research center, UPC Barcelona Tech., Spain. In Spring 2017 he did his internship at GEIRI North America. Currently he is working as a graduate research assistant for his Ph.D. at West Virginia University, USA. His research interests include grid oscillations studies, smart grids analytics, power system operations, analysis and control.

Zhe YU received his B.E. degree from Department of Electrical Engineering, Tsinghua University, Beijing, China in 2009, M.S. degree from Department of Electrical and Computer Engineering, Carnegie Mellon University, Pittsburgh, USA in 2010, and Ph.D. degree from the School of Electrical and Computer Engineering, Cornell University, Ithaca, USA in 2016. He joined Global Energy Interconnection Research Institute North America (GEIRI North America) in 2017. His current research interests include power system and smart grid, demand response, dynamic programming, data processing and optimization. 
Di SHI received the $\mathrm{Ph} . \mathrm{D}$. degree in electrical engineering from Arizona State University, Tempe, USA, in 2012. He currently leads the Advanced Power System Analytics Group at GEIRI North America, San Jose, USA. He has published over 60 journal and conference papers and hold 15 US patents/patent applications. He is an editor of IEEE Transactions on Smart Grid.

Zhiwei WANG received the B.S. and M.S. degrees in electrical engineering from Southeast University, Nanjing, China, in 1988 and 1991, respectively. He is President of GEIRI North America, San Jose, USA. His research interests include power system operation and control, relay protection, power system planning and WAMS.

Dawei SU currently is the Deputy Director of Grid Dispatch Center at Jiangsu Electrical Power Company in China. His research interests include power system operation and control, intelligent substations and WAMS.

Chunlei XU received the B.S. degree in electrical engineering from Shanghai Jiao Tong University, Shanghai, China, in 1999. He currently leads the Dispatching Automation department at Jiangsu Electrical Power Company in China. His research interests include power system operation and control and WAMS.
Sarika Khushalani SOLANKI received B.E. from V.N.I.T Nagpur University, India and M.E. degree from Mumbai University, India in 1998 and 2000 respectively. She received the Ph.D. degree in Electrical and Computer Engineering from Mississippi State University, USA in 2006. She is currently an Assistant Professor in Lane Department of Computer Science and Electrical Engineering at West Virginia University, Morgantown, since August 2009. Prior to that, she worked for Open Systems International Inc, Minneapolis, as a Senior Engineer for three years. Her research interests include smart grid, computer applications, power system analysis and power system control.

Jignesh M. SOLANKI received B.E. from V.N.I.T, Nagpur, India and M.E. degree from Mumbai University, India in 1998 and 2000 respectively. He received the Ph.D. degree in Electrical and Computer Engineering from Mississippi State University, USA in 2006. He has been Research Assistant Professor in Lane Department of Computer Science and Electrical Engineering at West Virginia University, Morgantown, WV, since August 2009. Prior to that, he worked for Open Systems International Inc, Minneapolis, as a Senior Engineer for three years. His research interests include smart grid, multi-agent applications in and power system control. 